\title{
EFFECTS OF SLEEP DEPRIVATION, NICOTINE, AND SELENIUM ON WOUND HEALING IN RATS
}

\author{
KENAN GÜMÜŞTEKIN \\ Department of Physiology \\ Medical School, Atatürk University \\ Erzurum, Turkey \\ BEDRI SEVEN
}

Department of Nuclear Medicine

Medical School, Atatürk University

Erzurum, Turkey

\section{NEZIHE KARABULUT}

Nursing High School, Atatürk University

Erzurum, Turkey

ÖMER AKTAŞ

Department of Physiology, Medical School

Atatürk University

Erzurum, Turkey

\section{NESRIN GÜRSAN}

Department of Pathology

Medical School, Atatürk University

Erzurum, Turkey 


\section{S3AHIN ASLAN}

Department of Emergency Medicine

Medical School, Atatürk University

Erzurum, Turkey

\section{MUSTAFA KELES,}

Department of Internal Medicine

Medical School, Atatürk University

Erzurum, Turkey

\section{ERHAN VAROGLU}

Department of Nuclear Medicine

Medical School, Atatürk University

Erzurum, Turkey

\section{ŞENOL DANE}

Department of Physiology

Medical School, Atatürk University

Erzurum, Turkey

Effects of sleep deprivation (SD), nicotine, and selenium (Se) on wound healing were studied in 50 male rats (Sprague-Dawley strain). Fullskin-thickness burns were produced in animals. Then, SD, nicotine, and Se administrations were applied to animals in different groups. Wound healing was assessed by pathological analysis of wound by counting fibroblasts, capillary vessels, polymorphonuclear leucocytes (PNLs), and by measuring radiolabeled immunoglobulin $G(\operatorname{Ig} G)$ amount in wound area by radio-pharmaceutical and immunoscintigraphic procedures. The number of fibroblasts and capillary vessels were higher in control and Se groups than in sleep deprivation and nicotine groups, and the number of PNLs and the radiolabeled polyvalent IgG levels were higher in $S D$ and nicotine groups than in control and Se groups. The results suggest that $S D$ and nicotine may delay wound healing and that Se supplementation may accelerate wound healing by preventing nicotineinduced oxidative stress and lipid peroxidation.

Keywords nicotine, selenium, sleep deprivation, wound healing

Acute phase response is defined as a pathological condition induced by many factors, for example, infection, inflammation, tissue damage, neoplastic growth, or immunological disorders (Saitoh et al., 2002). 
The acute phase response includes haemodynamic changes, PNL infiltration, and release of inflammatory mediators. PNL infiltration is a constant feature of inflammation.

Sleep is thought to be essential for recovery from injury, and lost or disturbed sleep is believed to hinder post-injury wound healing (tissue repair) (Landis \& Whitney, 1997). Disrupted or lost sleep is a common experience in the immediate postoperative period (Aurell \& Elmquist, 1985; Fontaine, 1989), and patients often express concern about losing sleep after surgery (Knapp-Spooner \& Yarcheski, 1992; Simpson et al., 1996a, 1996b). The consequences of lost sleep are of particular concern to nurses and other clinicians who provide or guide care for patients after acute surgical or accidental injury and are in a position to facilitate or educate patients about sleep. Questions regarding the possible effects of sleep loss on tissue repair and mechanisms by which sleep loss might negatively affect wound healing have been raised (Lee \& Stotts, 1990).

Nicotine, a major toxic component of cigarette smoke, induces oxidative stress both in vitro and in vivo. Increased free radical production and cooperative lipid peroxidation levels in pancreatic tissue of rats incubated with nicotine (Wetscher et al., 1995), and increased lipid peroxidation in Chinese hamster ovary cells incubated with nicotine and smokeless tobacco extract (Yildiz et al., 1999) have been reported. In addition, elevated oxidative DNA damage in various tissues of mice exposed to side-stream cigarette smoke (Howard et al., 1998) and increased lipid peroxidation levels in tissues of intra-peritoneal nicotine administered rats (Helen et al., 2000) have been found. Furthermore, increased lipid peroxidation in blood of smokers (Kalra et al., 1991; Altuntas et al., 2002) and in blood and brain of nicotine administered rats (Suleyman et al., 2002; Gumustekin et al., 2003) have been reported.

Selenium is an essential micronutrient and small amounts of it are needed for tissue oxygenation and for protection against lipid peroxidation. It is involved in one of the main antioxidant defense systems of the body with the GSH-Px, which is the main plasma selenoprotein consisting of four identical subunits each containing one covalently bound Se atom (Gül et al., 2000).

The purpose of this article was to investigate whether Se administration has any beneficial effects on hindering effects of sleep deprivation and nicotine administration on wound healing in rats. 


\section{MATERIALS AND METHODS}

Five male rats (Sprague-Dawley strain with a body weight of $215 \pm$ $27 \mathrm{~g}$ ), fed with standard laboratory chow and water, were used in the study. They were obtained from the Physiology Laboratory, Medical Faculty of Atatürk University All groups were homogeneous. The animals were randomly divided into 5 groups (10 rats per group) and placed in separate cages during the study. The groups were as follows:

Group I: control

Group II: sleep deprivation (SD)

Group III: SD + Se (4 ppm in drinking water)

Group IV: SD + nicotine $(0.5 \mathrm{mg} / \mathrm{kg} /$ day, i.p. $)$

Group V: SD + nicotine $+\mathrm{Se}$

Animal experimentations were carried out in an ethically proper way by following guidelines as set by the Ethical Committee of the Atatürk University.

Animals in groups III and $\mathrm{V}$ received Se (4 ppm in drinking water) p.o. pre-burn for 10 days.

Hydrogen tartrate salt of nicotine (Sigma N-5260) was dissolved in $0.9 \% \mathrm{NaCl}$ solution to get a $0.15 \mathrm{mg} / \mathrm{ml}$ concentration of nicotine. Then $\mathrm{pH}$ of the nicotine solution was adjusted to 7.4 by $0.1 \mathrm{~N}$ $\mathrm{NaOH}$. Nicotine $(0.5 \mathrm{mg} / \mathrm{kg} / \mathrm{d})$ was administered by intra-peritoneal injection to groups IV and V pre-bum for 10 days.

Full-skin-thickness burns were produced by the method of Arons' burn model (Arons, 1965). Under light ether anaesthesia, rats were injected intra-peritoneally with ketamin- $\mathrm{HCl}$ (Ketalar®, $20 \mathrm{mg} / \mathrm{kg}$ ). After careful shaving of the surface of the gluteal region, each rat was exposed to heated metal apparatus with a diameter of $1 \mathrm{~cm}$ $\left(90^{\circ} \mathrm{C}, 20 \mathrm{~s}\right)$. Thus, a second-degree burn was formed after nicotine and selenium administration for 10 days.

SD was carried out by the standard platform method (Vogel, 1975). For the 72-h sleep loss period, water to a depth of $2.5 \mathrm{~cm}$ replaced the wood shavings in the cages of the sleep-deprived rats. Using this method, the size of the platform permits some non-rapid eye movement (NREM) sleep, but each time the rat is about to 
enter REM sleep, loss of postural muscle tone occurs, balance is lost, and the rat wakes up as it begins to fall toward the water in the bottom of the cage. Landis reported $>35 \%$ loss of slow wave sleep, and $>80 \%$ loss of REM sleep during a 72- to 96-h period of sleep loss using this method (Landis, 1996). SD was used for 3 days in groups II, III, IV, and V after wound application. Control animals were maintained in cages with small platforms and wood shavings after wound application.

A total amount of $185 \mathrm{MBq}(5 \mathrm{mCi}) \mathrm{Tc}-99 \mathrm{~m} \mathrm{IgG}$, was injected via the tail vein after finishing 3 sleepless days. Anaesthetized animals were placed under the gamma camera equipped with low energy, general purpose, and parallel-hole collimator. Anterior whole body images and spot views of the wound areas were taken at 4th $\mathrm{h}$ after injection. The energy level of the gamma camera peaked at $140 \mathrm{keV}$ and a $20 \%$ energy window was selected. For semiquantitative analysis, regions of interest (ROI) were drawn around the wound areas and wound-free region for calculating background activity. The target to background ratio for each images was calculated.

The specimens from animals were harvested after $72 \mathrm{~h}$ of sleep deprivation under deep anaesthesia immediately after immunoscintigraphic procedure. The specimens were fixed in $10 \%$ formalin and routinely processed for paraffin embedding. From each sample, $4 \mu \mathrm{m}$ thick sections were obtained. Standard histological procedures with hematoxylin and eosin stains were used to prepare sections for identifying the extent of infiltration of PNLs, fibroblasts, and proliferation of small blood vessel. A semi-quantitative evaluation scale with scores from 0 to 3 (no cells to innumerable/extensive cells) was used to evaluate the extent of PNLs, fibroblasts, and proliferation of small blood vessels in samples. This scale is routinely and widely used for histological evaluation of tissue in wound healing models (Pierce et al., 1988).

\section{RESULTS}

There were significant positive correlations between the number of fibroblasts with the number of capillary vessels $(r=.84, p<.001)$ 
and negative correlation with the number of PNLs $(r=.67, p<$ $.001)$. There were significant negative correlations between the number of capillary vessels with the number of PNLs $(r=.67, p<.001)$ and the radiolabeled polyvalent IgG levels $(r=.33, p<.05)$. There were significant positive correlations between the number of PNLs and the radiolabeled polyvalent IgG levels $(r=.32, p<.05)$.

Table 1 presents the means and standard deviations of the numbers of fibroblasts, capillary vessels, and PNLs and the radiolabeled polyvalent IgG levels of animals in different groups. In one-way ANOVA, the differences among groups were statistically significant for all parameters. Post hoc LSD test was used for comparisons of groups.

The numbers of fibroblasts and capillary vessels were higher in control group than in SD group $(p<.001)$. In addition, the number of PNLs $(p<.001)$ and the radiolabeled polyvalent IgG levels $(p<$ .05) were higher in SD group than in control group.

The numbers of fibroblasts and capillary vessels were higher in control group than in SD + nicotine group $(p<.001)$. In addition, the number of PNL $(p<.001)$ and the radiolabeled polyvalent IgG levels $(p<.05)$ were higher in SD + nicotine group than in control group.

There were no differences in the numbers of fibroblasts, capillary vessels, and PNL between SD and SD + nicotine groups. But the radiolabeled polyvalent $\mathrm{IgG}$ levels were higher in $\mathrm{SD}+$ nicotine group than in $\mathrm{SD}$ group $(p<.01)$.

The numbers of fibroblasts $(p<.05)$ and capillary vessels $(p<$ $.001)$ were higher in SD + Se group than in SD group. The number of PNLs $(p<.001)$ and the radiolabeled polyvalent IgG levels $(p<$ $.01)$ were higher in SD group than in SD + Se group.

TABLE 1. The means and standard deviations of the numbers of fibroblasts, capillary vessels, and PNLs, and the radiolabeled polyvalent IgG levels of animals in different groups

\begin{tabular}{lccccccc}
\hline $\begin{array}{l}\text { Groups } \\
\text { Parameters }\end{array}$ & Control & $S D$ & $S D+S e$ & $\begin{array}{c}S D+ \\
\text { nicotine }\end{array}$ & $\begin{array}{l}S D+S e \\
\text { nicotine }\end{array}$ & $F_{4,35}$ & $p$ \\
\hline Fibroblast & $2.6 \pm 0.69$ & $0.6 \pm 0.69$ & $1.2 \pm 0.42$ & $0.3 \pm 0.48$ & $2.6 \pm 0.52$ & 35.88 & $<0.001$ \\
Capillary & $2.6 \pm 0.69$ & $0.3 \pm 0.48$ & $1.4 \pm 0.52$ & $0.1 \pm 0.32$ & $2.6 \pm 0.52$ & 53.29 & $<0.001$ \\
PNL & $0.2 \pm 1.42$ & $2.5 \pm 0.53$ & $0.7 \pm 0.82$ & $2.6 \pm 0.52$ & $0.5 \pm 0.53$ & 39.79 & $<0.001$ \\
IgG & $4.4 \pm 2.75$ & $6.6 \pm 2.61$ & $3.5 \pm 0.76$ & $9.22 \pm 2.8$ & $7.76 \pm 1.1$ & 11.43 & $<0.001$ \\
\hline
\end{tabular}


The number of fibroblasts and capillary vessels were higher in control group than in SD + Se group $(p<.001)$. But there were no differences in the number of PNLs and the radiolabeled polyvalent IgG levels between these two groups.

The numbers of fibroblasts and capillary vessels were higher in $\mathrm{SD}+$ nicotine + Se group than in $\mathrm{SD}+$ nicotine group (fibroblast: $p<.001$; capillary vessels: $p<.001)$. In addition, the number of $\mathrm{PNL}$ was higher in SD + nicotine group than in $\mathrm{SD}+$ nicotine $+\mathrm{Se}$ group $(p<.001)$. But there was no difference in the radiolabeled polyvalent IgG levels between these two groups.

\section{DISCUSSION}

In this study, positive correlations were found between the number of fibroblasts with the number of capillary vessels and negative correlation with the number of PNLs, however, negative correlations were found between the number of capillary vessels with the number of PNLs and the radiolabeled polyvalent IgG levels. A positive correlation was found between the number of PNLs and the radiolabeled polyvalent IgG levels. The earlier correlations indicate that the number of fibroblasts and capillary vessels shows rapid wound healing, but the number of PNLs and the radiolabeled polyvalent IgG levels shows delayed wound healing.

In the present study, the numbers of fibroblasts and capillary vessels were higher in control group than in SD group, and the number of PNLs and the radiolabeled polyvalent IgG levels were higher in SD group than in control group. These results suggest that SD impairs wound healing. These findings are consistent with many studies (Evans \& French, 1995; Helm, 1992; Adam \& Oswald, 1984) but not a study performed by Landis and Whitney (1997). They claimed that SD does not impair cellular and biochemical indicators of tissue repair.

In the present study, the number of fibroblasts and capillary vessels were higher in control group than in SD + nicotine group; and the number of PNL and the radiolabeled polyvalent IgG levels were higher in SD + nicotine group than in control group. In addition, the radiolabeled polyvalent $\mathrm{IgG}$ levels were higher in $\mathrm{SD}+$ nicotine 
than in SD group. These results show that nicotine delays tissue repair. The cause of this effect may be due to nicotine-induced oxidative stress and lipid peroxidation (Kalra, et al., 1991; Altuntas et al., 2002; Suleyman et al., 2002; Gümüştekin et al., 2003).

In the present study, the number of fibroblasts and capillary vessels were higher in SD + Se group than in SD group. The number of PNLs and the radiolabeled polyvalent IgG levels were higher in SD group than in SD + Se group. The number of fibroblasts and capillary vessels were higher in control group than in SD + Se group. The number of fibroblasts and capillary vessels were higher in SD + nicotine + Se group than in SD + nicotine group. In addition, the number of PNLs was higher in SD + nicotine group than in SD + nicotine + Se group. Berger et al. (1992) and Berger et al. (1996) examined Se losses and balances in 10 patients with burns, and observed lower Se and plasma GSH-Px concentrations that could not be explained by measured losses. There are studies reporting increased GSH-Px activity in Se-supplemented L1210 cells cultured in fetal bovine serum (Lin et al., 1993; Geiger et al., 1991; Gül et al., 2000).

The present results indicate that supplementation of Se accelerates wound healing by preventing nicotine-induced oxidative stress. So, supplementations of Se at appropriate doses to subjects at high risk for oxidative damage, like surgery and burn, may have beneficial effects.

In conclusion, whereas SD and nicotine delayed wound healing, Se supplementation accelerated wound healing. Therefore, for speedy wound healing, patients with wound, burn, or applied operation must avoid smoking and SD, and Se supplementation may be useful for them.

\section{REFERENCES}

Adam, K., \& Oswald, I. (1984). Sleep helps healing. British Medical Journal, 289, 14001401.

Altuntas, I., Dane, S., \& Gümüstekin, K. (2002). Effects of cigarette smoking on lipid peroxidation. Journal of Basic and Clinical Physiology and Pharmacology, 13, 69-72.

Axons, M. S. (1965). Burn wound infection-A review. Connecticut Medicine, 29, 718 722. 
Aurell, J., \& Elmquist, D. (1985). Sleep in the surgical intensive care unit: Continuous polygraphic recording of sleep in nine patients receiving postoperative care. British Medical Journal, 290, 1029-1032.

Berger, M. M., Cavadini, C., Bart, A., Mansourian, R., Guinchard, S., Bartholdi, I., Vandervale, A., Krupp, S., Chiolero, R., \& Freeman J. (1992). Cutaneous copper and zinc losses in burns. Burns, 18, 373-380.

Berger, M. M., Cavadini, C., Chiolero, R., \& Dirren, H. (1996). Copper, selenium, and zinc status and balances after major trauma. Journal of Trauma, 40, 103-109.

Evans, J. C., \& French, D. G. (1995). Sleep and healing in intensive care settings. Dimensions of Critical Care Nursing, 14, 189-199.

Fontaine, D. K. (1989). Measurement of nocturnal sleep patterns in trauma patients. Heart \& Lung, 18, 402-410.

Geiger, P. G., Thomas, I. P., \& Girotti, A. W. (1991). Lethal damage to murine L1210 cells by exogenous lipid hydroperoxides: Protective role of glutathione-dependent selenoperoxidases. Archives of Biochemistry and Biophysics, 288, 671-680.

Gül, M., Temoçin, S., \& Hanninen, O. (2000). Selenium supplementation sensitizes renca cells to tert-butylhydroperoxide induced loss of viability. Indian Journal of Experimental Biology, 38, 1020-1025.

Gümüstekin, K., Altinkaynak, K., Timur, H., Taysi, S., Oztasan, N., Polat, M. F., Akcay, F., Suleyman, H., Dane, S., Gul, M. (2003). Vitamin E but not Hippophea rhamnoides L. prevented nicotine-induced oxidative stress in rat brain. Human and Experimental Toxicology, 22, 425-431.

Helen, A., Krishnakumar, K., Vijayammal, P. L., \& Augusti, K. T. (2000). Antioxidant effect of onion oil (Allium cepa. Linn) on the damages induced by nicotine in rats as compared to alpha-tocopheroi. Toxicology Letters, 116, 61-68.

Helm, P. A. (1992). Burn rehabilitation: Dimensions of the problem. Clinics in Plastic Surgery, 19, 551-559.

Howard, D. J., Briggs, L. A., \& Pritsos, C. A. (1998). Oxidative DNA damage in mouse heart, liver, and lung tissue due to acute side-stream tobacco smoke exposure. Archives of Biochemistry and Biophysics, 352, 293-297.

Kalra, J., Chaudhary, A. K., \& Prasad, K. (1991). Increased production of oxygen free radicals in cigarette smokers. International Journal of Experimental Pathology, 72, 1-7.

Knapp-Spooner, C., \& Yarcheski, A. (1992). Sleep patterns and stress in patients having coronary bypass. Heart \& Lung, 21, 342-349.

Landis, C. A. (1996). Altered sleep patterns with the platform method of REM sleep deprivation in rats. Journal of Sleep Research, 25, 469.

Landis, C. A., \& Whitney, J. D. (1997). Effects of 72 hours sleep deprivation on wound healing in the rat. Research in Nursing and Health, 20, 259-267.

Lee, K. A., \& Stotts, N. A. (1990). Support of the growth hormone-somatomedin system to facilitate healing. Heart \& Lung, 19, 157-164.

Lin, F., Thomas, J. P., \& Girotti, A. W. (1993). Hyperexpression of catalase in seleniumdeprived murine L1210 cells. Archives of Biochemistry and Biophysics, 305, 176-185.

Pierce, G. F., Mustoe, T. A., Senior, R. M., Reed, J., Griffin, G. L., Thomason, A., \& Deuel, T. F. (1988). In vivo incisional wound healing augmented by platelet-derived growth factor and recombinant c-sis gene homodimeric proteins. The Journal of Experimental Medicine, 167, 974-987.

Saitoh, T., Kokue, E., \& Shimoda, M. (2002). The impact of acute phase response on the plasma clearance of antipyrine, theophylline, phenytoin and nifedipine in rabbits. Journal of Veterinary Pharmacolology and Therapeutics, 23, 153-158.

Simpson, T., Lee, E. R., \& Cameron, C. (1996a). Relationships among sleep dimensions and factors that impair sleep after cardiac surgery. Research in Nursing and Health, 19, 213-223.

Simpson, T., Lee, E. R., \& Cameron, C. (1996b). Patients' perceptions of environmental factors that disturb sleep after cardiac surgery. American Journal of Critical Care, 5, $173-181$ 
Suleyman, H., Gümüştekin, K., Taysi, S., Keles, S., Öztasan, N., Aktas, Ö., Altinkaynak, K., Timur, H., Akcay, F., Akar, S., Dane, S., \& Gül, M. (2002). Beneficial effects of Hippophae rhamnoides L. on nicotine induced oxidative stress in rat blood compared with vitamin E. Biological and Pharmaceutical Bulletin, 25, 1133-1136.

Vogel, G. (1975). A review of REM sleep deprivation. Archives of General Psychiatry, 132, 749-761.

Yildiz, D., Liu, Y. S., Ercal, N., \& Armstrong, D. W. (1999). Comparison of pure nicotine- and smokeless tobacco extract-induced toxicities and oxidative stress. Archives of Environmental Contamination and Toxicology, 37, 434-439.

Wetscher, G. J., Bagchi, M., Bagchi, D., Perdikis, G., Hinder, P. R., \& Glaser, K. (1995). Free radical production in nicotine treated pancreatic tissue. Free Radical Biology and Medicine, 18, 877-882. 
Copyright of International Journal of Neuroscience is the property of Taylor \& Francis Ltd and its content may not be copied or emailed to multiple sites or posted to a listserv without the copyright holder's express written permission. However, users may print, download, or email articles for individual use. 